\title{
An expanding role for osimertinib for the treatment of ErbB family driven NSCLC
}

\author{
Anthony C. Faber ${ }^{1}$, Timothy F. Burns ${ }^{2,3}$ \\ ${ }^{1}$ Philips Institute for Oral Health Research, VCU School of Dentistry and Massey Cancer Center, Richmond, VA, USA; ${ }^{2}$ Department of \\ Pharmacology and Chemical Biology, University of Pittsburgh, Pittsburgh, PA, USA; ${ }^{3}$ Department of Medicine, Division of Hematology-Oncology, \\ UPMC Hillman Cancer Center, Pittsburgh, PA, USA \\ Correspondence to: Anthony C. Faber. Philips Institute for Oral Health Research, VCU School of Dentistry and Massey Cancer Center, Richmond, \\ VA, USA. Email: acfaber@vcu.edu. \\ Comment on: Liu S, Li S, Hai J, et al. Targeting HER2 Aberrations in Non-Small Cell Lung Cancer with Osimertinib. Clin Cancer Res 2018;24:2594-604.
}

Submitted Jul 31, 2018. Accepted for publication Aug 06, 2018.

doi: $10.21037 /$ tcr.2018.08.10

View this article at: http://dx.doi.org/10.21037/tcr.2018.08.10

The discovery that a subset of non-small cell lung cancers (NSCLC) were dependent upon reoccurring activating EGFR (ErbB1) mutations (1-4), primarily deletions of exon 19 or an activating point mutation, $L 858 R$, ushered in the era of targeted therapies for lung cancer. Shortly after, first-generation EGFR tyrosine kinase inhibitors (TKIs) that were capable of inhibiting these mutant proteins proved effective. However, the enthusiasm was hampered by the clinical appearance of acquired resistance mediated by a secondary EGFR mutation, converting threonine at position 790 to methionine (T790M) (5), in effect, blocking the ability of the inhibitor to access the ATP binding pocket of EGFR.

It was later determined that almost half the cases of EGFR inhibitor resistance are caused by the acquisition, or enrichment from a small percent of alleles present prior to initiation of treatment, of T790M (6). This led to the subsequent development of next-generation EGFR inhibitors, with third-generation EGFR inhibitors like osimertinib (AZD9291) demonstrating efficacy in EGFR mutant NSCLCs with acquired T790M mutations $(7,8)$. More recently, the FLAURA investigators compared osimertinib with $1^{\text {st }}$ generation EGFR TKIs (erlotinib and gefitinib) in treatment-naïve EGFR mutant advanced NSCLC (9). Strikingly, patients treated with osimertinib demonstrated a median duration of response of 17.2 months compared to 8.5 months for $1^{\text {st }}$ generation EGFR TKIs. Furthermore, there was an improved toxicity profile with osimertinib. There have been other impressive responses to osimertinib in the first-line therapy for patients with de novo T790M mutations as well, further demonstrating the potential of the drug (10). The FLAURA study has resulted in the adoption of osimertinib as the preferred first-line therapy for EGFR mutant lung cancer based on its improved efficacy and favorable toxicity profile. However, it remains to be known whether overall survival of these patients will exceed or fall short of those who receive firstline EGFR inhibitors until the acquisition of T790M, and then receive osimertinib.

\section{The role of other ErbB members in EGFR mutant NSCLC}

EGFR also known as ErbB1 is part of a four-member family of transmembrane proteins, along with ErbB2 (HER2), ErbB3, and ErbB4 (11). While EGFR is actionable in lung, glioma, head and neck and colorectal cancer, HER2 is a prominent drug target itself, particularly in breast cancer (12) and gastric cancer (13), but does have an established role in lung cancer as well. Interestingly, one of the earliest cell lines found to respond in vitro to gefitinib was NCI-H1819 (14), which is EGFR wild-type but HER2-dependent. In 175 lung adenocarcinomas, Li and colleagues (15) found that HER2 was both amplified and mutated at a rate of $\sim 3 \%$, and these alterations were found independent of each other. HER2 has also emerged as an important modulator of EGFR inhibitor response in cancer, including $E G F R$ amplified colorectal cancer and EGFR mutant NSCLC (16). Pao and colleagues demonstrated that HER2 is amplified in $\sim 10 \%$ 


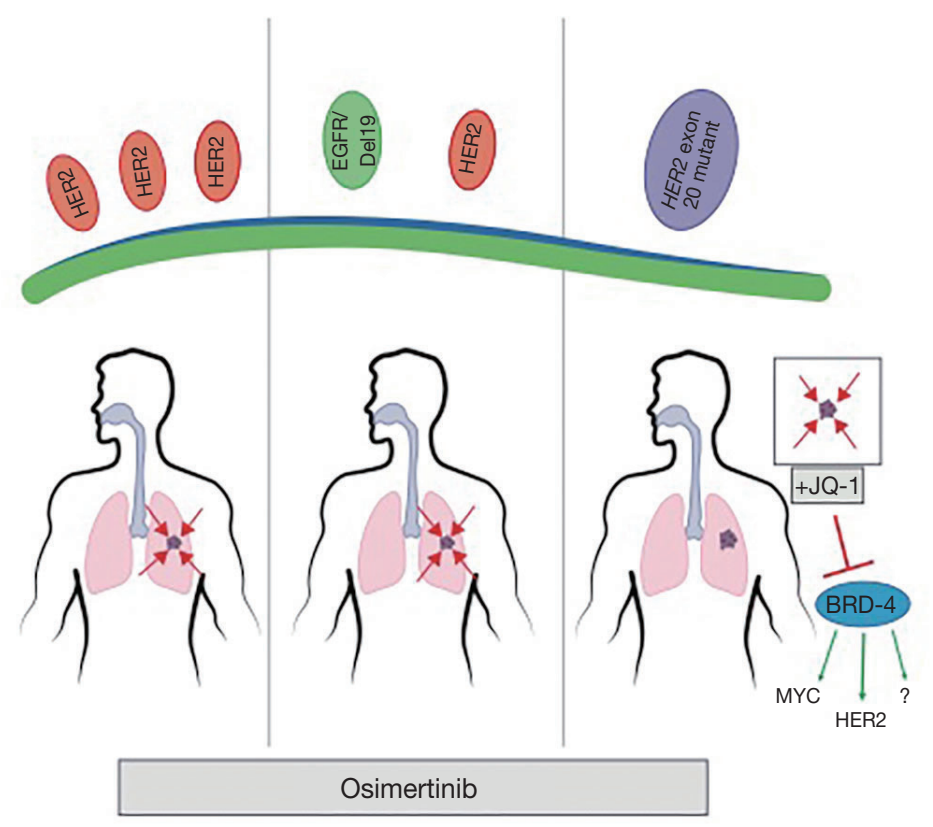

Figure 1 Genetically-distinct NSCLCs have sensitivity to osimertinib-based therapies in mouse models. NSCLC tumors are depicted with HER2 overexpression (left), HER2 overexpression coupled with an activating EGFR exon 19 deletion mutation (middle), or with HER2 exon deletion mutations (right). In the first two cases, these tumors respond well to osimertinib in GEMMs of NSCLC. While HER2 exon deletions are insensitive to single-agent osimertinib (right), these tumors are sensitized by the addition of the BET inhibitor, JQ-1, which inhibits bromodomain-containing proteins such as bromodomain-containing protein-4 (BRD4). This lead to decreased HER2 and MYC expression as well as other potential oncogenic drivers leading to synergistic activity of the combination in vivo.

of patients with acquired resistance to EGFR inhibitor, compared to $1 \%$ of EGFR inhibitor treatment-naïve $E G F R$ mutant NSCLCs (17). Furthermore, HER2 amplification was independent of T790M mutations in the acquired resistant tumors (17).

Clinically, responses of HER2-altered NSCLCs to both HER2 inhibitors and EGFR inhibitors have been mixed. De Grève reported the pan- and irreversible ErbB inhibitor afatinib led to objective responses in three out of three HER2 mutant metastatic NSCLC patients (18). Dacomitinib, a similar second generation pan-inhibitor of the ErbB family (other than ErbB3), resulted in 3 partial responses out of 26 patients (19). More recently, the HER2 antibody-drug conjugate, ado-trastuzumab emtansine (20), demonstrated a partial response rate of $44 \%$ in 18 patients, with a median survival of 5 months. Responses were seen in a number of different mutations, and also in patients with HER2 exon 20 insertions. While these responses were relatively short-lived, it is significant that both a large subset of patients responded across a broad range of HER2 alterations.
Despite the responses elicited by ado-trastuzumab emtansine (20), it is clear that a better understanding of the molecular and biological characteristics of the different HER2 alterations, both alone and in the presence of EGFR mutations, that are seen across the NSCLC landscape, would aid in finding the best available inhibitor to treat each NSCLC molecular subtype and hopefully result in more durable responses.

To this point, Liu and colleagues (21) directly tackled this question by testing discrete HER2 alterations in NSCLC and the therapeutic efficacy of osimertinib against each alteration in mouse models (Figure 1). The researchers tested osimertinib against three molecularly distinct HER2 altered NSCLCs: (I) HER2 overexpressed NSCLC; (II) EGFRdel19/HER2 overexpressed NSCLC; and (III) HER2 exon 20 insertion in mouse NSCLC models. The animal experiments were performed using several unique models of NSCLC genetically engineered mouse models (GEMMs) Therefore, these authors were uniquely positioned to be able to robustly study the biology and therapeutic responses of these cancer types. 
First however, the authors confirmed the increased efficacy of osimertinib against HER2 compared to other EGFR TKIs by demonstrated that the HER2 overexpressing $\mathrm{Ba} / \mathrm{F} 3$ cells had significantly lower GI50s $(10.4 \mathrm{nM})$, than erlotinib (438 $\mathrm{nM})$, underlying the ability of osimertinib to potently inhibit HER2. They next turned to their GEMM model of HER2 overexpressing NSCLC. Interestingly, the authors demonstrated that overexpressing HER2 was sufficient to drive high-grade adenocarcinoma formation. Importantly, these tumors uniformly shrank following osimertinib treatment but were resistant to either erlotinib or the pan-HER TKI, afatinib, demonstrating in vivo that osimertinib is uniquely effective against HER2 overexpressing NSCLC.

Next, the authors looked at the effects of HER2 overexpression on EGFR mutant deletion 19 tumors, a combination found in $\sim 10 \%$ of EGFR mutant NSCLC with acquired resistance to first generation TKIs (17). Again, they employed a unique GEMM model that both expressed the mutant EGFR and overexpressed HER2. While the survival of these mice was extended by 2 weeks with erlotinib, the survival of osimertinib-treated mice was 24 weeks, with several of these mice surviving the experiment. These data demonstrate that in the second tested NSCLC subtype, osimertinib was also active.

The authors then turned their attention to HER2 exon 20 mutant NSCLCs. First, by expressing the most frequent HER2 exon 20 insertion mutation (A775_G776insYVMA) in $\mathrm{Ba}-\mathrm{F} 3$ cells, the investigators demonstrated, similarly to the HER2-overexpressing $\mathrm{Ba} / \mathrm{F} 3$ cells, osimertinib was sufficient to inhibit these cells. However, the amount of osimertinib to reach GI50 levels was about 4 times greater than what was required in HER2 wt cells. These data proved to portend the results of osimertinib treatment in a GEMM of HER2 exon 20 mutant (A775_G776insYVMA) NSCLC, as these mice responded poorly to osimertinib and experience no improvement in survival. Interestingly, the investigators had previously demonstrated that afatinib, which was less effective than osimertinib in their HER2 overexpression model, was effective in the HER2 exon 20 mutant model alone or in combination with rapamycin (22). In is unclear, why these EGFR TKIs display HER2 alteration subtype efficacy but deserves further investigation. The lack of activity of HER 2 exon 20 mutant NSCLC is also consistent with a recent study demonstrated that the development of HER2 exon 20 mutations as mechanism of acquired resistance to osimertinib in patients with $E G F R$ mutant NSCLC (23).
The authors then asked, given the in vitro signal from osimertinib, would a rationally-implemented second drug be sufficient to sensitize osimertinib in the HER2 exon 20 deletion model. To inform of this second drug, the authors noted signaling changes following osimertinib treatment in one of the only HER2 mutant NSCLC cell linesNCI-H1781-which contains a rarer HER2 20 insertion mutation. They found a convergence in osimertinib inhibited signaling to two targets of the BET inhibitor JQ-1, p21 and c-MYC (24). The authors then tested the combination in the GEMM, and, impressively, found that while neither single-agent had significant activity, the combination led to profound survival increases in the mice.

Overall, the study by Wong and colleagues adds to the growing understanding that HER 2 can be a successfully druggable target in NSCLC, both in the absence and presence of $E G F R$ mutations. It also demonstrates the power that GEMMs afford to study cancers with relatively low frequencies and/or those with a lack of available established cell lines. Interestingly, some of these same authors recently demonstrated that while osimertinib is ineffective in HER 2 mutant NSCLCs harboring exon 20 mutations, the less structurally cumbersome pan-ErbB inhibitor poziotinib is effective (25). In combination with the promising clinical data emerging with ado-trastuzumab emtansine (20), there are now several FDA-approved agents, albeit some approved for other indications, which can now be tested in HER2 altered NSCLC subtypes.

Lastly, the authors demonstrate a rationale combination of osimertinib with the BET inhibitor, JQ-1, for exon 20 deletion HER2 NSCLCs. As the authors point out, this is also interesting for the reason that exon 20 deletion EGFR NSCLCs are structurally analogous, make up $4-10 \%$ of EGFR mutant NSCLCs, and are irresponsive to traditional EGFR inhibitors (26). Thus, testing the combination of osimertinib/JQ-1 in EGFR exon 20 deletion NSCLCs may be the next step in further delineating promising strategies to treat EGFR and HER2 altered NSCLCs. This study also raises the question of how BET inhibitors resensitize to osimertinib in HER2 exon 20 mutant NSCLC. Although the authors demonstrated that JQ1 treatment lead to decrease HER2 and MYC expression, it is still unknown whether if modulation of these pathways is responsible for the observed in vivo synergy between osimertinib and JQ1. Several recent studies have shown that Bromodomaincontaining protein-4 (BRD4), a key target of BET inhibitors, is critical for the upregulation of oncogenic RTKs in the setting of acquired resistance to PI3K, HER2, 
and EGFR inhibitors $(25,27,28)$. Therefore, future studies both in the laboratory and clinic will be necessary to define whether the critical target BET inhibitors when combined with a targeted agent is the primary RTK, a secondary or bypass RTK (such as MET), or downstream signaling pathway (such as MYC). These studies may not only lead to better treatment of HER2 altered NSCLC subtypes but may lead to novel upfront combinations for EGFR mutant NSCLC that may prevent or delay the development of resistance to osimertinib.

\section{Acknowledgments}

Funding: None.

\section{Footnote}

Provenance and Peer Review: This article was commissioned and reviewed by the Section Editor Yijiu Ren (Department of Thoracic Surgery, Shanghai Pulmonary Hospital, Tongji University School of Medicine, Shanghai, China).

Conflicts of Interest: Both authors have completed the ICMJE uniform disclosure form (available at http://dx.doi. org/10.21037/tcr.2018.08.10). The authors have no conflicts of interest to declare.

Ethical Statement: The authors are accountable for all aspects of the work in ensuring that questions related to the accuracy or integrity of any part of the work are appropriately investigated and resolved.

Open Access Statement: This is an Open Access article distributed in accordance with the Creative Commons Attribution-NonCommercial-NoDerivs 4.0 International License (CC BY-NC-ND 4.0), which permits the noncommercial replication and distribution of the article with the strict proviso that no changes or edits are made and the original work is properly cited (including links to both the formal publication through the relevant DOI and the license). See: https://creativecommons.org/licenses/by-nc-nd/4.0/.

\section{References}

1. Paez JG, Janne PA, Lee JC, et al. EGFR mutations in lung cancer: correlation with clinical response to gefitinib therapy. Science 2004;304:1497-500.

2. Sordella R, Bell DW, Haber DA, et al. Gefitinib- sensitizing EGFR mutations in lung cancer activate antiapoptotic pathways. Science 2004;305:1163-7.

3. Lynch TJ, Bell DW, Sordella R, et al. Activating mutations in the epidermal growth factor receptor underlying responsiveness of non-small-cell lung cancer to gefitinib. N Engl J Med 2004;350:2129-39.

4. Pao W, Miller V, Zakowski M, et al. EGF receptor gene mutations are common in lung cancers from "never smokers" and are associated with sensitivity of tumors to gefitinib and erlotinib. Proc Natl Acad Sci U S A 2004;101:13306-11.

5. Pao W, Miller VA, Politi KA, et al. Acquired resistance of lung adenocarcinomas to gefitinib or erlotinib is associated with a second mutation in the EGFR kinase domain. PLoS Med 2005;2:e73.

6. Piotrowska Z, Sequist LV. Treatment of EGFR-Mutant Lung Cancers After Progression in Patients Receiving First-Line EGFR Tyrosine Kinase Inhibitors : A Review. JAMA Oncol 2016;2:948-54.

7. Sequist LV, Piotrowska Z, Niederst MJ, et al. Osimertinib Responses After Disease Progression in Patients Who Had Been Receiving Rociletinib. JAMA Oncol 2016;2:541-3.

8. Jänne PA, Yang JC, Kim DW, et al. AZD9291 in EGFR inhibitor-resistant non-small-cell lung cancer. N Engl J Med 2015;372:1689-99.

9. Soria JC, Ohe Y, Vansteenkiste J, et al. Osimertinib in Untreated EGFR-Mutated Advanced Non-Small-Cell Lung Cancer. N Engl J Med 2018;378:113-25.

10. Ancevski Hunter K, Friedland DM, Villaruz LC, et al. First-Line Osimertinib in Patients with Treatment-Naive Somatic or Germline EGFR T790M-Mutant Metastatic NSCLC. J Thorac Oncol 2018;13:e3-5.

11. Sharma SV, Settleman J. ErbBs in lung cancer. Exp Cell Res 2009;315:557-71.

12. Floros KV, Lochmann TL, Hu B, et al. Coamplification of miR-4728 protects HER2-amplified breast cancers from targeted therapy. Proc Natl Acad Sci U S A 2018;115:E2594-603.

13. Bang YJ, Van Cutsem E, Feyereislova A, et al. Trastuzumab in combination with chemotherapy versus chemotherapy alone for treatment of HER2-positive advanced gastric or gastro-oesophageal junction cancer (ToGA): a phase 3, open-label, randomised controlled trial. Lancet 2010;376:687-97.

14. Amann J, Kalyankrishna S, Massion PP, et al. Aberrant epidermal growth factor receptor signaling and enhanced sensitivity to EGFR inhibitors in lung cancer. Cancer Res 2005;65:226-35. 
15. Li BT, Ross DS, Aisner DL, et al. HER2 Amplification and HER2 Mutation Are Distinct Molecular Targets in Lung Cancers. J Thorac Oncol 2016;11:414-9.

16. Yonesaka K, Zejnullahu K, Okamoto I, et al. Activation of ERBB2 signaling causes resistance to the EGFRdirected therapeutic antibody cetuximab. Sci Transl Med 2011;3:99ra86.

17. Takezawa K, Pirazzoli V, Arcila ME, et al. HER2 amplification: a potential mechanism of acquired resistance to EGFR inhibition in EGFR-mutant lung cancers that lack the second-site EGFRT790M mutation. Cancer Discov 2012;2:922-33.

18. De Grève J, Teugels E, Geers C, et al. Clinical activity of afatinib (BIBW 2992) in patients with lung adenocarcinoma with mutations in the kinase domain of HER2/neu. Lung Cancer 2012;76:123-7.

19. Kris MG, Camidge DR, Giaccone G, et al. Targeting HER2 aberrations as actionable drivers in lung cancers: phase II trial of the pan-HER tyrosine kinase inhibitor dacomitinib in patients with HER2-mutant or amplified tumors. Ann Oncol 2015;26:1421-7.

20. Li BT, Shen R, Buonocore D, et al. Ado-Trastuzumab Emtansine for Patients With HER2-Mutant Lung Cancers: Results From a Phase II Basket Trial. J Clin Oncol 2018;36:2532-7.

21. Liu S, Li S, Hai J, et al. Targeting HER2 Aberrations in Non-Small Cell Lung Cancer with Osimertinib. Clin Cancer Res 2018;24:2594-604.

Cite this article as: Faber AC, Burns TF. An expanding role for osimertinib for the treatment of ERbB family driven NSCLC. Transl Cancer Res 2018;7(Suppl 7):S787-S791. doi: 10.21037/tcr.2018.08.10
22. Perera SA, Li D, Shimamura T, et al. HER2YVMA drives rapid development of adenosquamous lung tumors in mice that are sensitive to BIBW2992 and rapamycin combination therapy. Proc Natl Acad Sci U S A 2009; 106:474-9.

23. Ramalingam SS, Yang JC, Lee CK, et al. Osimertinib As First-Line Treatment of EGFR Mutation-Positive Advanced Non-Small-Cell Lung Cancer. J Clin Oncol 2018;36:841-9.

24. Delmore JE, Issa GC, Lemieux ME, et al. BET bromodomain inhibition as a therapeutic strategy to target c-Myc. Cell 2011;146:904-17.

25. Robichaux JP, Elamin YY, Tan Z, et al. Mechanisms and clinical activity of an EGFR and HER2 exon 20-selective kinase inhibitor in non-small cell lung cancer. Nat Med 2018;24:638-46.

26. Yasuda H, Park E, Yun CH, et al. Structural, biochemical, and clinical characterization of epidermal growth factor receptor (EGFR) exon 20 insertion mutations in lung cancer. Sci Transl Med 2013;5:216ra177.

27. Stuhlmiller TJ, Miller SM, Zawistowski JS, et al. Inhibition of Lapatinib-Induced Kinome Reprogramming in ERBB2-Positive Breast Cancer by Targeting BET Family Bromodomains. Cell Rep 2015;11:390-404.

28. Stratikopoulos EE, Dendy M, Szabolcs M, et al. Kinase and BET Inhibitors Together Clamp Inhibition of PI3K Signaling and Overcome Resistance to Therapy. Cancer Cell 2015;27:837-51. 